\title{
Comparing the Rates and the Activation Parameters for the Forward Reaction between the Triplet State of Zinc Cytochrome $c$ and Cupriplastocyanin and the Back Reaction between the Zinc Cytochrome $c$ Cation Radical and Cuproplastocyanin ${ }^{\dagger}$
}

\author{
Maja M. Ivković-Jensen,,${ }^{\ddagger}$ G. Matthias Ullmann," Milan M. Crnogorac, ${ }^{\ddagger}$ Mikael Ejdebäck, ${ }^{\perp}$ Simon Young, ${ }^{\perp}$ \\ Örjan Hansson, ${ }^{\perp}$ and Nenad M. Kostić*, \\ Department of Chemistry, Iowa State University, Ames, Iowa 50011, Institut für Kristallographie, Freie Universität Berlin, \\ Takustrasse 6, 14195 Berlin, Germany, and Department of Biochemistry and Biophysics, Lundberg Institute, \\ Göteborg University and Chalmers University of Technology, S-41390 Göteborg, Sweden
}

Received July 16, 1998; Revised Manuscript Received November 17, 1998

\begin{abstract}
This is a comparative study of the photoinduced (so-called forward) electron-transfer reaction ${ }^{3} \mathrm{Zncyt} / \mathrm{pc}(\mathrm{II}) \rightarrow \mathrm{Zncyt}^{+} / \mathrm{pc}(\mathrm{I})$, between the triplet state of zinc cytochrome $c$ ( ${ }^{3} \mathrm{Zncyt}$ ) and cupriplastocyanin [pc(II)], and the thermal (so-called back) electron-transfer reaction Zncyt ${ }^{+} / \mathrm{pc}(\mathrm{I}) \rightarrow \mathrm{Zncyt} / \mathrm{pc}(\mathrm{II})$, between the cation (radical) of zinc cytochrome $c\left(\mathrm{Zncyt}^{+}\right)$and cuproplastocyanin [pc(I)], which follows it. Both reactions occur between associated (docked) reactants, and the respective unimolecular rate constants are $k_{\mathrm{F}}$ and $k_{\mathrm{B}}$. Our previous studies showed that the forward reaction is gated by a rearrangement of the diprotein complex. Now we examine the back reaction and complare the two. We study the effects of temperature (in the range $273.3-302.9 \mathrm{~K}$ ) and viscosity (in the range $1.00-17.4 \mathrm{cP}$ ) on the rate constants and determine enthalpies $\left(\Delta H^{\ddagger}\right)$, entropies $\left(\Delta S^{\ddagger}\right)$, and free energies $\left(\Delta G^{\ddagger}\right)$ of activation. We compare wild-type spinach plastocyanin, the single mutants Tyr83Leu and Glu59Lys, and the double mutant Glu59Lys/Glu60Gln. The rate constant $k_{\mathrm{B}}$ for wild-type spinach plastocyanin and its mutants markedly depends on viscosity, an indication that the back reaction is also gated. The activation parameters $\Delta H^{*}$ and $\Delta S^{\ddagger}$ show that the forward and back reactions have similar mechanisms, involving a rearrangement of the diprotein complex from the initial binding configuration to the reactive configuration. The rearrangements of the complexes ${ }^{3} \mathrm{Zncyt} / \mathrm{pc}$ (II) and $\mathrm{Zncyt}^{+} / \mathrm{pc}$ (I) that gate their respective reactions are similar but not identical. Since the back reaction of all plastocyanin variants is faster than the forward reaction, the difference in free energy between the docking and the reactive configuration is smaller for the back reaction than for the forward reaction. This difference is explained by the change in the electrostatic potential on the plastocyanin surface as $\mathrm{Cu}(\mathrm{II})$ is reduced to $\mathrm{Cu}(\mathrm{I})$. It is the smaller $\Delta H^{\ddagger}$ that makes $\Delta G^{\ddagger}$ smaller for the back reaction than for the forward reaction.
\end{abstract}

Site-directed mutagenesis is indispensable in the study of association and subsequent electron-transfer reactions between metalloproteins. When this method of molecular biology is combined with methods of chemical kinetics and with analysis of electrostatic interactions between metalloproteins, mechanisms of reactions can be analyzed at the molecular level. This study is one such analysis.

The observed rate of an electron-transfer reaction may correspond to the electron-transfer step or to some other process that is slower than this step. In the latter case, this rate-limiting process gates the electron transfer $(1-9)$. There is also a possibility that a fast but unfavorable equilibrium precedes the electron transfer; this unfavorable equilibrium

\footnotetext{
$\doteqdot$ This work was supported by the National Science Foundation through Grant MCB-9222741.

$\doteqdot$ Iowa State University.

$\S$ Present address: Department of Microbiology, University of Iowa, Iowa City, IA 52242.

"Freie Universität Berlin.

${ }^{\perp}$ Göteborg University and Chalmers Institute of Technology.
}

is said to be coupled with the electron transfer $(1,9)$. The proteins we investigate are plastocyanin (pc) ${ }^{1}$ and cytochrome $c$ (cyt). Both of them are fully characterized (10-14). Our group and others have shown that the replacement of iron(II) in cytochrome $c$ by zinc(II) does not perturb the conformation of the protein $(15,16)$ and its association with other metalloproteins (17-21). Both cytochrome $c$ and zinc cytochrome $c$ bind to plastocyanin through their basic patch surrounding the exposed heme edge. Plastocyanin binds to both cytochrome $c$ and zinc cytochrome $c$ with its broad acidic patch (22-26). Ultraviolet/visible, CD, EPR, and NMR spectra and reduction potentials of the plastocyanin mutants show that the overall conformation and the copper site are not detectably perturbed by mutation of surface

\footnotetext{
${ }^{1}$ Abbreviations: cyt, cytochrome $c$; cyt(III), ferricytochrome $c$; cyt(II), ferrocytochrome $c$; pc, plastocyanin; pc(I), cuproplastocyanin; pc(II), cupriplastocyanin; Zncyt, zinc cytochrome $c$; ${ }^{3}$ Zncyt, triplet (exited) state of zinc cytochrome $c$; $\mathrm{Zncyt}^{+}$, cation radical of zinc cytochrome $c$.
} 


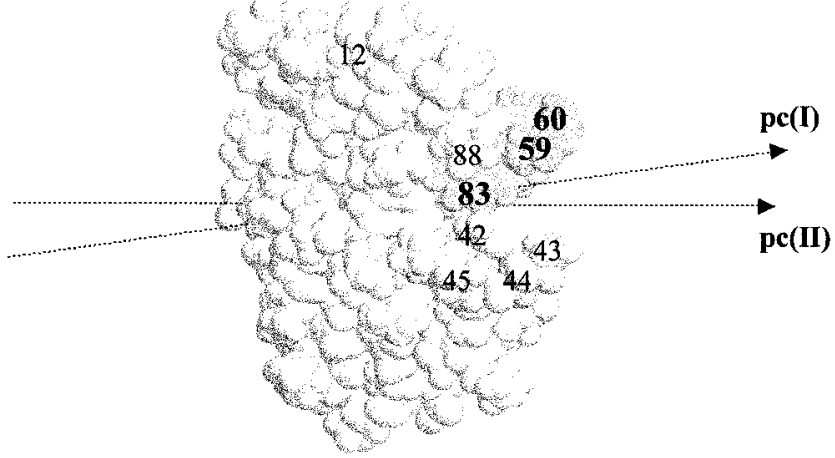

FIGURE 1: Structure of wild-type spinach plastocyanin. Positions of mutated residues (Glu59, Glu60, and Tyr83) and of other relevant residues (Leu12, Asp42, Glu43, Asp44, Glu45, and Gln88) are highlighted, and the dipole-moment vectors of pc(II) and pc(I) are shown. This figure was generated with the program RasMol.

residues in the acidic patch (27-32). Our group and others have used mutants of plastocyanin to study its reactions with photosystem I $(27,30,33-37)$, with cytochrome $f(28,29$, $38-40)$, and with cytochrome $c(28,29,41,42)$.

Previous studies of the quenching of the triplet state ${ }^{3}$ Zncyt by cupriplastocyanin $(26,43-54)$ showed that the intracomplex reaction in eq 1 (forward reaction) could be observed at low ionic strength, at which the two proteins associate to a great extent. The intracomplex reaction in eq 2 (back reaction) could also be observed by monitoring of the cation radical $\mathrm{Zncyt}^{+}(44)$.

$$
\begin{gathered}
{ }^{3} \mathrm{Zncyt} / \mathrm{pc}(\mathrm{II}) \stackrel{k_{\mathrm{F}}}{\longrightarrow} \mathrm{Zncyt}^{+} / \mathrm{pc}(\mathrm{I}) \\
\mathrm{Zncyt}^{+} / \mathrm{pc}(\mathrm{II}) \stackrel{k_{\mathrm{B}}}{\longrightarrow} \mathrm{Zncyt} / \mathrm{pc}(\mathrm{II})
\end{gathered}
$$

The dynamic properties of many diprotein complexes in solution were observed (55-65). The drastic decrease of the rate constant $k_{\mathrm{F}}$ as solution viscosity increases show that $k_{\mathrm{F}}$ corresponds to a structural rearrangement of the diprotein complexes between zinc cytochrome $c$ and cupriplastocyanin. This process was investigated by analysis of electrontunneling paths $(66,67)$, by changing experimental conditions $(53,54)$, and by site-directed mutagenesis $(41,42)$. We determined the activation parameters $\Delta H^{\ddagger}$ and $\Delta S^{\ddagger}$ for the rate of rearrangement, $k_{\mathrm{F}}$, and interpreted these activation parameters in terms of molecular surfaces (68) of the two proteins. We proposed the pathway of this rearrangement on the basis of the activation parameters obtained with 14 variants of plastocyanin (42). Since only the mutants that have lysine in positions 59 and 88 have considerable effect on the activation parameters, the pathway of rearrangement of the diprotein complex ${ }^{3} \mathrm{Zncyt} / \mathrm{pc}$ (II) does not involve residues 12, 42, and 43 and involves residues 59 and 88 (Figure 1).

In this work, we explore the back electron-transfer reaction in eq 2 . The rate constant $k_{\mathrm{B}}$ for this unimolecular reaction is $1.1 \times 10^{6} \mathrm{~s}^{-1}$ for zinc cytochrome $c$ and French-bean plastocyanin at $298 \mathrm{~K}(44)$. Under the same conditions the rate constant $k_{\mathrm{F}}$ for the preceding forward electron-transfer reaction in eq 1 is $2.5 \times 10^{5} \mathrm{~s}^{-1}$. Because the back reaction is only a little faster than the forward reaction, it can be observed (44). We now explore the back reaction for wildtype spinach plastocyanin; for its mutants that showed a marked decrease in the rate of the forward reaction,
Glu59Lys and Glu69Lys/Glu60Gln; and for the mutant Tyr83Leu, which we have not studied before and which is interesting because residue 83 sits between the two acidic clusters and also between positions 59 and 88. The dependence of the rate constant $k_{\mathrm{B}}$ on temperature and viscosity can reveal the mechanism of the back electron transfer.

\section{MATERIALS AND METHODS}

Chemicals. Distilled water was demineralized to a resistivity greater than $17 \mathrm{M} \Omega \cdot \mathrm{cm}$. Chromatography gels (CM Sephadex C-50, Sephadex G-25 and G-75, and Sephadex DEAE A-25) were purchased from Sigma Chemical Co. Hydrogen fluoride, nitrogen, and ultrapure argon were purchased from Air Products Co. All other chemicals were purchased from Fisher Chemical Co.

Buffers. Sodium dihydrogen phosphate and sodium monohydrogen phosphate were used to make buffers with ionic strength of $2.5 \mathrm{mM}$ and $\mathrm{pH} 7.0$.

Viscosity. The absolute viscosity $(\eta)$ of water and of aqueous solutions of glycerol at different temperatures were taken from tables $(69-71)$. The relative viscosity $\left(\eta / \eta_{0}\right)$ of buffers with and without glycerol was measured with a thermostated glass viscometer; the absolute error was \pm 0.05 $\mathrm{cP}$. Given $\eta_{0}=1.002 \mathrm{cP}$, the absolute viscosity was calculated. Both calculations and experiments showed the contribution of salts to viscosity to be negligible. The contribution of proteins, at micromolar concentrations, is negligible.

Zinc Cytochrome $c$. Horse-heart cytochrome $c$ was purchased from Sigma Chemical Co. The iron-free (so-called free-base) form was made, purified, and reconstituted with zinc(II) by a modification (21) of the original procedure (17, 18). The product, zinc cytochrome $c$, was handled at $4{ }^{\circ} \mathrm{C}$, in the dark. The criteria of purity were the absorbance ratios $A_{423} / A_{549}>15.4$ and $A_{549} / A_{585}<2.0$ and the rate constant for natural decay of the triplet state, $k_{\mathrm{d}}<110 \mathrm{~s}^{-1}$.

Plastocyanin. Recombinant wild-type protein from spinach and mutants of it were prepared by the published method (72) and purified chromatographically (41). All proteins were desalted, transferred into a $2.5 \mathrm{mM}$ phosphate buffer at $\mathrm{pH}$ 7.0, and stored in liquid nitrogen. Before each series of kinetic experiments, plastocyanin was treated with a small excess of dissolved $\mathrm{K}_{3}\left[\mathrm{Fe}(\mathrm{CN})_{6}\right]$, which was then removed with Centricon ultrafiltration cells. Concentrations of the proteins were determined from their UV-vis spectra, on the basis of the known absorptivities: $\epsilon_{423}=2.43 \times 10^{5} \mathrm{M}^{-1}$ $\mathrm{cm}^{-1}$ for zinc cytochrome $c(18)$ and $\epsilon_{597}=4700 \mathrm{M}^{-1} \mathrm{~cm}^{-1}$ for all the mutants of cupriplastocyanin (72).

Flash Kinetic Spectrophotometry. So-called laser flash photolysis on the microsecond scale was done with a standard apparatus (44). Argon was passed first through water and then through the buffered solution to be deaerated. The required volume of the buffer was deaerated in a $10-\mathrm{mm}$ cuvette for $30 \mathrm{~min}$. The cell jacket was connected to a $30-\mathrm{L}$ circulating bath, Forma 2067, which maintained the temperature within $\pm 0.2^{\circ} \mathrm{C}$. The actual temperature in the cell was calibrated with an Omega HH82 digital thermometer and was known with a precision of $\pm 0.1^{\circ} \mathrm{C}$. The temperature range was 273.3-302.9 K. After the temperature of the buffer was adjusted, other solutions were added. The concentration of zinc cytochrome $c$ was always $10.0 \mu \mathrm{M}$. 
After each exposure to air, the solution in the cuvette was gently deaerated for $10-30 \mathrm{~min}$, longer for more viscous solutions. Determinations of $k_{\mathrm{d}}$ in control experiments proved this deaeration to be thorough enough. Formation and decay (natural or by quenching) of the triplet state, ${ }^{3} \mathrm{Zncyt}$, were monitored at $460 \mathrm{~nm}$. Its concentration depended on the intensity of the laser pulse and was ca. $1.0 \mu \mathrm{M}$. Formation and disappearance of the cation radical, $\mathrm{Zncyt}^{+}$, were monitored at $675 \mathrm{~nm}$. At each set of conditions (temperature and glycerol concentration), 5-10 laser pulses were delivered. Error bars in the figures enclose all the corresponding experimental values. Experiments on the nanosecond scale were done with a Q-switched Nd-YAG laser described elsewhere (73). Handling of the sample was different. Cuvette was cooled in the ice bath, and as it was slowly returning to the room temperature, the solution temperature was directly measured with an Omega HH82 digital thermometer. The temperature range was $277.7-297.0 \mathrm{~K}$.

Kinetics. The rate constants were obtained from the changes in the absorbances at 460 and $675 \mathrm{~nm}$ with time. The absorbance decrease at $460 \mathrm{~nm}$ corresponds to the disappearance of the ${ }^{3} \mathrm{Zncyt}$, described by eq 3 . The fractional contributions of the two phases (subscripts 1 and 2) are described by eq 4 . The $k_{1}, k_{2}, f_{1}$, and $f_{2}$ values obtained are

$$
\begin{gathered}
\Delta A_{460}=a_{1} \exp \left(-k_{1} t\right)+a_{2} \exp \left(-k_{2} t\right)+b \\
f_{1}=\frac{a_{1}}{a_{1}+a_{2}} \quad f_{2}=\frac{a_{2}}{a_{1}+a_{2}} \\
\Delta A_{675}=\Delta A_{\text {triplet }}+\Delta A_{\text {cation }} \\
\Delta A_{\text {triplet }}=a_{\mathrm{t}}\left[f_{1} \exp \left(-k_{1} t\right)+f_{2} \exp \left(-k_{2} t\right)\right] \\
\Delta A_{\text {cation }}=a_{\mathrm{c}}\left[\exp \left(-k_{3} t\right)-f_{1} \exp \left(-k_{1} t\right)-f_{2} \exp \left(-k_{2} t\right)\right]
\end{gathered}
$$

$$
\begin{aligned}
\Delta A_{\text {cation }}=a_{\mathrm{c}}\left[f_{1} \exp \left(-k_{3} t\right)+\right. & f_{2} \exp \left(-k_{4} t\right)- \\
& \left.f_{1} \exp \left(-k_{1} t\right)-f_{2} \exp \left(-k_{2} t\right)\right]
\end{aligned}
$$

used in the subsequent fittings. We chose experimental conditions such that the faster phase was dominant $\left(f_{1} \geq\right.$ $0.9, f_{2} \leq 0.1$ ), because we are interested in the unimolecular reaction. The change in absorbance at $675 \mathrm{~nm}$ is caused by $\mathrm{Zncyt}^{+}$(more) and by ${ }^{3} \mathrm{Zncyt}$ (less) and is described by eqs $5-8$. The wavelength $675 \mathrm{~nm}$ was chosen because there the difference between the absorbances of $\mathrm{Zncyt}^{+}$and ${ }^{3} \mathrm{Zncyt}$ is greatest. The contribution of the ${ }^{3}$ Zncyt to the change in absorbance at $675 \mathrm{~nm}$ is described by eq 6 , in which $a_{\mathrm{t}}$ is the instantaneous absorbance after the laser flash. The contribution of the $\mathrm{Zncyt}^{+}$to the change in absorbance at $675 \mathrm{~nm}$ can be described by eq 7, in which only the unimolecular $\left(k_{3}\right)$ back reaction is considered; or by eq 8 , in which both unimolecular $\left(k_{3}\right)$ and bimolecular $\left(k_{4}\right)$ back reactions are considered. The rise of the absorbance at 675 $\mathrm{nm}$ is due to the back reaction, and the fall of this absorbance is due to the forward reaction; the fall matches the rate obtained by monitoring ${ }^{3} \mathrm{Zncyt}$ at $460 \mathrm{~nm}$ (44). The parameters $k_{1}, k_{2}, f_{1}, f_{2}$, and $a_{\mathrm{t}}$ were fixed; the fitting to eqs 5,6 , and 7 (together) gave the parameters $a_{\mathrm{c}}$ and $k_{3}$, whereas the fitting to eqs 5,6 , and 8 (together) gave the three parameters $a_{\mathrm{c}}, k_{3}$, and $k_{4}$. The two methods of fitting, at our experimental conditions, gave values of $k_{3}$ that were the same, within the standard errors of fitting. The rate constant $k_{3}$ is the unimolecular rate constant of interest, $k_{\mathrm{B}}$ in eq 1 . The traces were analyzed with kinetic software from Olis, Inc., and with the fitting program SigmaPlot 1.02, from Jandel Scientific Co. The standard errors of fitting, which are given in the tables, are computed by dividing the standard deviation by the square root of the number of measurements.

Energies and Geometrical Properties of the Two Diprotein Complexes in Four Configurations. We examined four configurations of the electrostatic complex between ferrocytochrome $c$ and cupriplastocyanin, as a model for ${ }^{3} \mathrm{Zncyt} /$ $\mathrm{pc}(\mathrm{II})$, and four configurations of the complex between ferricytochrome $c$ and cuproplastocyanin, as a model for $\mathrm{Zncyt}^{+} / \mathrm{pc}$ (II). The structures of horse-heart ferrocytochrome (1hrc) (74) and of poplar cupriplastocyanin (1plc) (75) were obtained from the Brookhaven Protein Data Bank. Hydrogen atoms were added with CHARMM (76). The structure of spinach cupriplastocyanin was obtained by "mutating" the nonidentical residues. The conformation of each new residue was matched to that of the original residue. The structure was then minimized with fixed atoms that had not changed upon "mutation" and unconstrained new atoms that had no counterparts in the structure of poplar plastocyanin. Dipole moments were calculated with respect to the center of mass (77), assuming the usual $\mathrm{p} K_{\mathrm{a}}$ values at $\mathrm{pH} 7$ and CHARMM22 (78) partial charges for all atoms. The structures of horseheart cytochrome $c$ and of spinach plastocyanin were superimposed on the proteins in these three configurations of the cyt/pc electrostatic complex by using the Kabsch algorithm (79). In each configuration, the energies of monopole-monopole, monopole-dipole, and dipole-dipole interactions and the total Coulombic energy were calculated by standard formulas $(80,42)$. The energy of monopolar interaction was obtained from eq 9 , the energies of monopoledipole interactions were obtained from eqs 10 and 11, the energy of dipolar interaction was obtained from eq 12, and the total electrostatic energy was obtained from eq 13. The

$$
\begin{gathered}
E_{Z Z}=\frac{\mathrm{e}^{2}}{4 \pi \epsilon \epsilon_{0}} \frac{Z_{1} Z_{2}}{r} \\
E_{P Z}=\frac{\mathrm{e}^{2}}{4 \pi \epsilon \epsilon_{0}} \frac{P_{1} Z_{2} \cos \theta_{1}}{r^{2}} \\
E_{Z P}=\frac{\mathrm{e}^{2}}{4 \pi \epsilon \epsilon_{0}} \frac{Z_{1} P_{2} \cos \theta_{2}}{r^{2}} \\
E_{P P}=\frac{\mathrm{e}^{2}}{4 \pi \epsilon \epsilon_{0}} \frac{P_{1} P_{2}}{r^{3}}\left(2 \cos \theta_{1} \cos \theta_{2}+\sin \theta_{1} \sin \theta_{2} \cos \phi\right) \\
E=E_{Z Z}+E_{P Z}+E_{Z P}+E_{P P}
\end{gathered}
$$

symbols in eqs $9-13$ have their usual meanings: $\epsilon$ is permittivity of water, $\epsilon_{0}$ is permittivity of vacuum, $Z_{1}$ and $Z_{2}$ are the respective net charges at $\mathrm{pH} 7$ of cytochrome $c$ and plastocyanin, $P_{1}$ is the dipole moment of cytochrome $c$, $P_{2}$ is the dipole moment of plastocyanin, $\theta_{1}$ and $\theta_{2}$ are the angles in the respective proteins between the positive end of dipole moment and the vector that connects the center of 
Table 1: Rate Constants and Binding Constants

\begin{tabular}{lcccc}
\hline pc(II) species & $\begin{array}{c}k_{\mathrm{F}}{ }^{a} \times 10^{-5} \\
\left(\mathrm{~s}^{-1}\right)\end{array}$ & $\begin{array}{c}k_{\mathrm{B}}{ }^{b} \times 10^{-5} \\
\left(\mathrm{~s}^{-1}\right)\end{array}$ & $\begin{array}{c}K_{\mathrm{a}}{ }^{c} \times 10^{-5} \\
\left(\mathrm{M}^{-1}\right)\end{array}$ & $\begin{array}{c}K_{\mathrm{b}}{ }^{d} \times 10^{-5} \\
\left(\mathrm{M}^{-1}\right)\end{array}$ \\
\hline wild type & 2.3 & $9.6^{e}$ & $9 \pm 2^{f}$ & 23 \\
Glu59Lys & 0.26 & $0.35^{g}$ & $0.6 \pm 0.1^{f}$ & 2.1 \\
$\begin{array}{c}\text { Glu59Lys/ } \\
\quad \text { Glu60Gln }\end{array}$ & 0.20 & $0.29^{g}$ & $0.2 \pm 0.1^{f}$ & 0.73 \\
Tyr83Leu & 2.3 & $12.6^{e}$ & $1.2 \pm 0.5$ & 24 \\
\hline
\end{tabular}

${ }^{a}$ Rate constant for the unimolecular forward reaction in eq 1 at 298.2 $\mathrm{K}$; error is $\pm 10 \% .{ }^{b}$ Rate constant for the unimolecular back reaction in eq 2; error is $\pm 15 \% .{ }^{c}$ Experimental binding constant between cupriplastocyanin and zinc cytochrome $c .{ }^{d}$ Calculated binding constant between cuproplastocyanin and zinc cytochrome $c$ cation radical. ${ }^{e}$ At 297.0 K. ${ }^{f}$ From ref $42 .{ }^{g}$ At $298.2 \mathrm{~K}$.

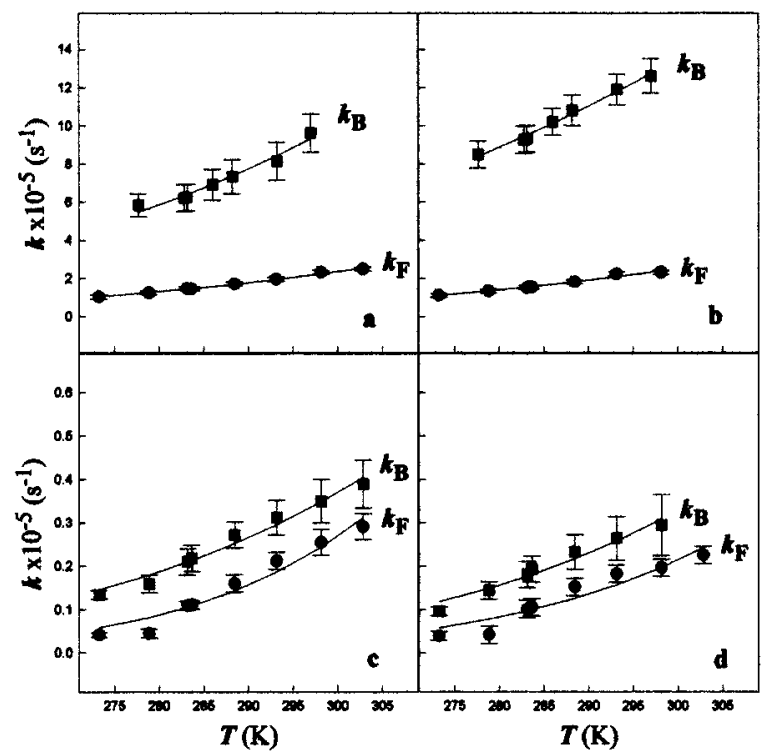

FiguRE 2: Dependence on temperature of the unimolecular rate constants $(k)$ for the intracomplex reactions in eq $1\left(k_{\mathrm{F}}, \bigcirc\right)$ and in eq $2\left(k_{\mathrm{B}}, \mathbf{D}\right)$, in sodium phosphate buffer at $\mathrm{pH} 7.0$ and ionic strength $2.5 \mathrm{mM}$ involving the following variants of spinach plastocyanin: (a) wild type, (b) Tyr83Leu, (c) Glu59Lys, and (d) Glu59Lys/ Glu60Gln. The lines are fittings to eq 14 . The $k_{\mathrm{F}}$ values in panels $\mathrm{a}, \mathrm{c}$, and $\mathrm{d}$ are from ref 42 .

mass of one protein with the center of mass of the other, $\phi$ is the torsion angle defined by the dipole moments and the centers of mass of both proteins, and $r$ is the distance between the two centers of mass. The configurations A, B, C, and D all have $\phi=0^{\circ}$; A has $\theta_{1}=0^{\circ}$ and $\theta_{2}=180^{\circ}$; $\mathrm{B}$ has $\theta_{1}=$ $30^{\circ}$ and $\theta_{2}=180^{\circ} ; \mathrm{C}$ has $\theta_{1}=30^{\circ}$ and $\theta_{2}=167^{\circ}$; and $\mathrm{D}$ has $\theta_{1}=30^{\circ}$ and $\theta_{2}=121^{\circ}$.

\section{RESULTS}

Reactions with Plastocyanin Variants. Effects of temperature on the reaction in eq 2 were studied at an ionic strength of $2.5 \mathrm{mM}$ and at $\mathrm{pH}$ 7.0. Wild-type plastocyanin and the Tyr83Leu mutant were studied on both microsecond and nanosecond time scales, while Glu59Lys and Glu59Lys/ Glu60Gln were studied on the microsecond time scale. The results are shown in Table 1 and Figure 2, together with those for the reaction in eq 1 (42). The fitting of the rate constants to the Eyring equation (eq 14) yielded the activation

$$
k_{\mathrm{F}}, k_{\mathrm{B}}=\frac{k_{\mathrm{Bol}} T}{h} \exp \frac{\Delta S^{\ddagger}}{R} \exp \frac{-\Delta H^{\ddagger}}{R T}
$$

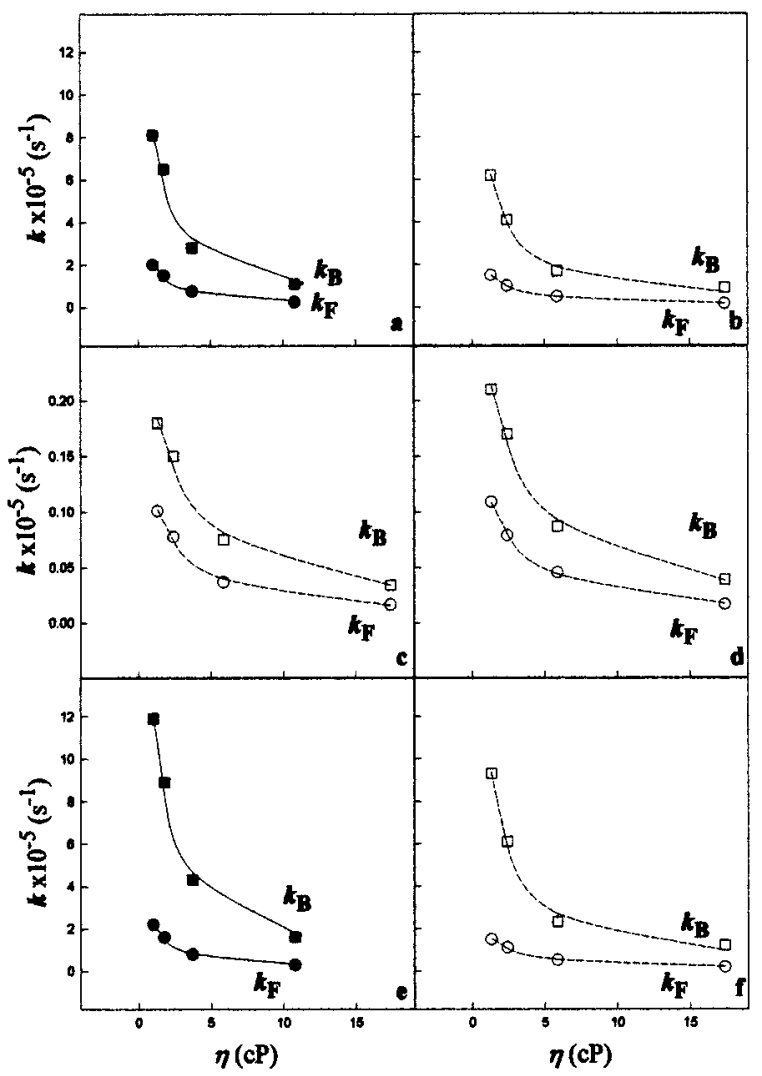

FIGURE 3: Dependence on viscosity of the unimolecular rate constants $(k)$ for the intracomplex reaction in eq $1\left(k_{\mathrm{F}}, \bigcirc\right.$ or $\left.\bigcirc\right)$ and in eq $2\left(k_{\mathrm{B}}, \boldsymbol{\square}\right.$ or $\left.\square\right)$, in sodium phosphate buffer at $\mathrm{pH} 7.0$ and ionic strength $2.5 \mathrm{mM}$, and temperatures specified (note the filled and open symbols), involving the following variants of spinach plastocyanin: (a) wild type at $293.2 \mathrm{~K}$, (b) wild type at $283.2 \mathrm{~K}$, (c) double mutant Glu59Lys/Glu60Gln at $283.2 \mathrm{~K}$, (d) Glu59Gln at $283.2 \mathrm{~K}$, (e) Tyr83Leu at $293.2 \mathrm{~K}$, and (f) Tyr83Leu at $283.2 \mathrm{~K}$. The lines are fittings to eq 15 . The $k_{\mathrm{F}}$ values in panels a-d are from ref 42 .

$$
k_{\mathrm{F}}, k_{\mathrm{B}}=\frac{k_{\mathrm{Bol}} T}{h} \frac{1+\sigma}{\eta+\sigma} \exp \left(\frac{-\Delta G^{\ddagger}}{R T}\right)
$$

parameters for the reactions in eqs 1 and 2 with different variants of plastocyanin; these parameters are listed in Table 2 . Viscosity effects on the reaction in eq 2 were studied at an ionic strength of $2.5 \mathrm{mM}$ and at $\mathrm{pH} 7.0$. The viscosity was changed at 283.2 and $293.2 \mathrm{~K}$ for wild-type plastocyanin and Tyr83Leu and at 283.2 $\mathrm{K}$ for the mutants Glu59Lys and Glu59Lys/Glu60Gln. The results are shown in Figure 3, together with those for the reaction in eq 1 (42). The fittings of the rate constants to eq 15 gave the results listed in Table 2 .

\section{DISCUSSION}

Forward and Back Reactions. Zinc cytochrome $c$ and plastocyanin exist in solution both free and bound to each other (Scheme 1). The concentration of the diprotein complex depends on the concentrations of both proteins, the ionic strength, and the variant of plastocyanin. The photoexcitation can occur in both the unassociated and the associated zinc cytochrome $c$. The forward bimolecular reaction occurs between free triplet zinc cytochrome $c,{ }^{3} \mathrm{Zncyt}$, and free cupriplastocyanin, pc(II). The forward unimolecular reaction occurs within the complex ${ }^{3} \mathrm{Zncyt} / \mathrm{pc}$ (II). The back unimolecular reaction can occur within the complex $\mathrm{Zncyt}^{+} / \mathrm{pc}(\mathrm{I})$. 


\begin{tabular}{|c|c|c|c|c|c|c|c|}
\hline pc species & & $\Delta H^{\ddagger}(\mathrm{kJ} / \mathrm{mol})$ & $\Delta S^{\ddagger}[\mathrm{J} /(\mathrm{mol} \mathrm{K})]$ & $T(\mathrm{~K})$ & $\sigma(\mathrm{cP})$ & $\Delta G^{\ddagger}(\mathrm{kJ} / \mathrm{mol})$ & $k_{0} \times 10^{-5}\left(\mathrm{~s}^{-1}\right)$ \\
\hline \multirow{4}{*}{ wild type } & forward & $18.1 \pm 0.7$ & $-82 \pm 3$ & 293.2 & $0.7 \pm 0.3$ & $41.9 \pm 0.1$ & 5.1 \\
\hline & back & $17 \pm 2$ & $-75 \pm 6$ & & $0.8 \pm 0.5$ & $38.5 \pm 0.2$ & 19 \\
\hline & forward & & & 283.2 & $0.9 \pm 0.1$ & $40.8 \pm 0.1$ & 3.7 \\
\hline & back & & & & $0.7 \pm 0.1$ & $37.4 \pm 0.2$ & 18 \\
\hline \multirow[t]{2}{*}{ Glu59Lys } & forward & $37 \pm 5$ & $-38 \pm 2$ & 283.2 & $1.8 \pm 0.2$ & $47.1 \pm 0.1$ & 0.19 \\
\hline & back & $21 \pm 2$ & $-86 \pm 6$ & & $2.2 \pm 0.5$ & $45.5 \pm 0.1$ & 0.35 \\
\hline \multirow[t]{2}{*}{ Glu59Lys/Glu60Gln } & forward & $31 \pm 6$ & $-60 \pm 2$ & 283.2 & $1.6 \pm 0.4$ & $47.2 \pm 0.1$ & 0.19 \\
\hline & back & $24 \pm 3$ & $-80 \pm 9$ & & $2.3 \pm 0.6$ & $45.9 \pm 0.2$ & 0.29 \\
\hline \multirow[t]{4}{*}{ Tyr83Leu } & forward & $18 \pm 2$ & $-80 \pm 6$ & 293.2 & $0.6 \pm 0.2$ & $41.7 \pm 0.1$ & 6.0 \\
\hline & back & $12 \pm 1$ & $-86 \pm 3$ & & $0.7 \pm 0.3$ & $37.6 \pm 0.1$ & 30 \\
\hline & forward & & & 283.2 & $1.2 \pm 0.3$ & $40.8 \pm 0.1$ & 3.2 \\
\hline & back & & & & $0.5 \pm 0.3$ & $36.4 \pm 0.2$ & 34 \\
\hline
\end{tabular}

${ }^{a}$ Activation parameters $\Delta H^{\ddagger}$ and $\Delta S^{\ddagger}$ were obtained by fitting of the rate constants $k_{\mathrm{F}}$ (from ref 42 ) and $k_{\mathrm{B}}$ (from this work) to the Eyring equation (eq 14); protein friction and free energy of activation $\Delta G^{\ddagger}$ were obtained by fitting of the rate constants $k_{\mathrm{F}}$ (from ref 42 ) and $k_{\mathrm{B}}$ (from this work) to eq 15; and rate constants $k_{0}$ at zero viscosity were obtained by extrapolation of eq 15 .

Scheme 1

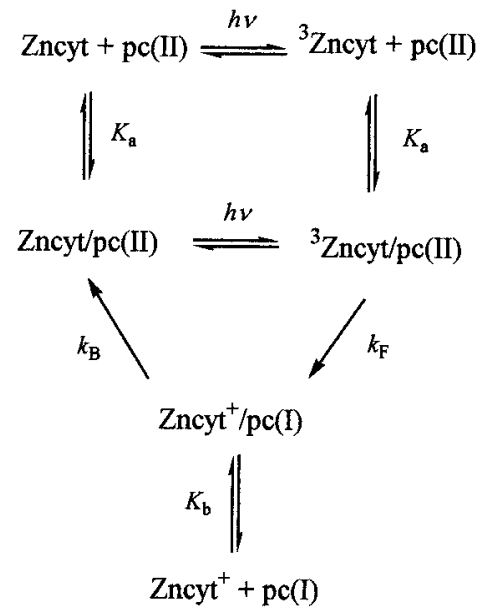

If the complex dissociates, the back bimolecular reaction occurs between free zinc cytochrome $c$ cation radical, $\mathrm{Zncyt}^{+}$, and free cuproplastocyanin, pc(I). The constant $K_{\mathrm{a}}$ is the association constant between zinc cytochrome $c$ and cupriplastocyanin, and the constant $K_{\mathrm{b}}$ is the association constant between the zinc cytochrome $c$ cation radical and cuproplastocyanin. The association constant $K_{\mathrm{a}}$ is probably equal to, or somewhat smaller than, the association constant $K_{\mathrm{b}}$, since the product of charges $Z_{1} Z_{2}$ is greater for the cation radical and cuproplastocyanin $(-63)$ than for the triplet zinc cytochrome $c$ and cupriplastocyanin (-48); in both of these products the $Z_{2}$ value is that of wild-type plastocyanin. Thus, when the fractional contribution of the faster phase for the forward reaction is larger than 0.9 , the fractional contribution of the faster phase for the back reaction also is larger than 0.9 . Under these conditions, we can precisely study the unimolecular reactions of interest, those shown in eqs 1 and 2.

Association of the Proteins. While the concentration of zinc cytochrome $c$ was always $10.0 \mu \mathrm{M}$, the concentration of cupriplastocyanin was varied to adjust the fractional contribution of the faster phase for the forward reaction, $f_{1}$. For $f_{1}>0.9$, the concentrations of wild-type cupriplastocyanin, the mutant Tyr83Leu, the mutant Glu59Lys, and the mutant Glu59Lys/Gly60Gln had to be at least 40, 70, 80, and $90 \mu \mathrm{M}$ respectively. The required excess of cupriplastocyanin depends on the magnitude of the association constant $K_{\mathrm{a}}$ for the particular variant of plastocyanin (Table
1). The $K_{\mathrm{a}}$ values for zinc cytochrome $c$ and the variant were determined from the fractional contributions of the two phases by using eq 16

$$
\begin{aligned}
& \frac{a_{1}}{a_{1}+a_{2}}=\frac{1}{2[\mathrm{Zncyt}]_{0}}\left\{[\mathrm{Zncyt}]_{0}+[\mathrm{pc}(\mathrm{II})]_{0}+K_{\mathrm{a}}^{-1}-\right. \\
& \left.\left(\left([\mathrm{Zncyt}]_{0}+[\mathrm{pc}(\mathrm{II})]_{0}+K_{\mathrm{a}}^{-1}\right)^{2}-4[\mathrm{Zncyt}]_{0}[\mathrm{pc}(\mathrm{II})]_{0}\right)^{1 / 2}\right\}
\end{aligned}
$$

(three values in Table 1 are from ref 42). The values of $K_{\mathrm{b}}$, for zinc cytochrome $c$ cation radical and cuproplastocyanin, are similar to or larger than the corresponding values of $K_{\mathrm{a}}$; see the argument about $f_{1}$ above. The values of $K_{\mathrm{b}}$ are calculated by using the correlation between the free energy of association and calculated Coulombic energy. We have previously determined experimental values of $K_{\mathrm{a}}$ for wildtype and 12 mutants of plastocyanin (42). We can then calculate the free energies of association $\Delta G_{\mathrm{a}}$ (eq 17) and plot them vs theoretical Coulombic energy $E$ in eq 13 . We find a simple linear correlation between $\Delta G_{\mathrm{a}}$ and $E$ that we use to estimate $\Delta G_{\mathrm{b}}$ from $E$, assuming that the free energies of association are related to Coulombic energies in the same manner (eq 18). Several assumptions made in the estimation

$$
\begin{gathered}
\Delta G_{\mathrm{a}}=-R T \ln K_{\mathrm{a}} \\
\Delta G_{\mathrm{a}, \mathrm{b}}=C_{1} E+C_{0} \\
K_{\mathrm{b}}=\exp \left(-\Delta G_{\mathrm{b}} / R T\right)
\end{gathered}
$$

of $K_{\mathrm{b}}$ make these values in Table 1 very approximate. The estimate of $K_{\mathrm{b}}$ for Tyr83Leu is probably much larger than the actual value, because $K_{\mathrm{a}}$ is lowered upon mutation of Tyr83 to Leu even though the charge does not change.

Plastocyanin Mutants. While the forward reaction of the wild-type cupriplastocyanin can be followed on the microsecond time scale, the back reaction of the wild-type cuproplastocyanin must be followed on the nanosecond time scale. The forward reaction of the wild-type plastocyanin was studied with both the microsecond and the nanosecond laser, and the results agreed well. Mutations in position 59 and 88 most affect the forward rate constant, $k_{\mathrm{F}}(42)$. Preliminary experiments have shown that the back reactions of the mutants Glu59Lys/Glu60Gln and Glu59Lys, which differ the most from the wild-type plastocyanin, can be 
studied with the microsecond laser. The back reaction of the mutant Tyr83Leu, which behaves similarly to the wild-type protein, had to be studied with the nanosecond laser as well.

Effect of Mutation in Position 83 on the Rate Constant $k_{F}$. Tyrosine 83 is a highly conserved residue in higher plant plastocyanins. Its role, however, is not clear. The mutation to leucine has an effect on the bimolecular rate constant for the ground-state (thermal) reaction with cytochrome $c(28)$ but has no effect on the bimolecular rate constant for the thermal reaction with photosystem I, a physiological partner (30). The bimolecular rate constants are difficult to interpret because they depend on protein association and the subsequent electron-transfer step. Moreover, any rearrangement or some other dynamic process is difficult to detect and study on the basis of composite rate constants. Although the reactions in eqs 1 and 2 are irrelevant to the biological role of plastocyanin, their study brings out the dynamic processes that may well be relevant to this role. By keeping the ionic strength low, at $2.5 \mathrm{mM}$, we favor the formation of the diprotein complex and directly observe the unimolecular process. Mutation to phenylalanine and to histidine does not affect the rate constant $k_{\mathrm{F}}(41)$. We find that the unimolecular rate constants $k_{\mathrm{F}}$ at all temperatures, and thus also the activation parameters, are the same for the wild-type cupriplastocyanin and the mutant Tyr83Leu. In the ground-state reaction Tyr83Leu had a lower rate then wild-type plastocyanin, so it was concluded that electron-transfer involves Tyr83 (28). The observed rate in the photoinduced reaction in eq 1 is the rate of rearrangement, a process that evidently does not involve Tyr83.

Effects of Temperature and of Plastocyanin Mutation on the Rate Constant $k_{B}$. Figure 2 shows the temperature dependence of the rate constants $k_{\mathrm{F}}$ (eq 1) and $k_{\mathrm{B}}$ (eq 2) for wild-type plastocyanin and its mutants. The driving forces for the two reactions are calculated from the potentials of the half-reactions. It is possible that the driving force have some temperature-dependent contributions other than temperature-dependent changes of redox potentials; but the potentials themselves do not significantly change within the temperature range of interest (81). The change of the driving force over the temperature range studied is no more than $\pm 2 \mathrm{~kJ} / \mathrm{mol}$. Because redox potentials of these (and other) metalloproteins depend only slightly on temperature, we can assume that so does the potential of ${ }^{3}$ Zncyt. Although the driving forces for the forward and back reactions are around -120 and around $-40 \mathrm{~kJ} / \mathrm{mol}$, respectively, the back reaction is always faster than the forward reaction. If these reactions were true electron transfer, we would expect the back reaction to be slower than the forward reaction, since their driving forces are too small for the Marcus inverted regime $(82,83)$. That the back reaction is always faster is the first indication that the back reaction is not simply electron transfer.

Effects of Viscosity and of Plastocyanin Mutation on the Rate Constant $k_{B}$. If the back reaction is independent of viscosity, then it is a true or coupled electron-transfer. If the back reaction is dependent on viscosity, it is a structural rearrangement. As Figure 3 shows, for wild-type cuproplastocyanin and for its mutants the rate constant for the reaction in eq 2 depends on viscosity. This dependence is consistent with gated electron transfer and not with true electron transfer. Because the equilibrium properties are not affected by solution viscosity (84), a fast preequilibrium coupled to electron transfer should not be affected by viscosity. Thus the observed rate, $k$, may be that of a slow rearrangement, $k_{\mathrm{r}}$, or the rate of a slow rearrangement with the contribution from the faster electron-transfer step, $k_{\mathrm{ET}}$ (eq 20). Effects of

$$
\frac{1}{k}=\frac{1}{k_{\mathrm{r}}}+\frac{1}{k_{\mathrm{ET}}}
$$

solution viscosity on the protein reactions have been studied $(84-88)$. We have shown that it is viscosity, and not the other properties of solutions, that determines the observed rates $(49,54)$. As Table 2 shows, the free energy of activation is always higher for the forward reaction than for the back reaction. This difference is more pronounced for the wildtype plastocyanin and the mutant Tyr83Leu than for the mutants Glu59Lys and Gly59Lys/Glu60Gln. We conclude that in the last two mutants the electrostatic potential of plastocyanin is less sensitive to the oxidation state of copper. We will test this conclusion with theoretical calculations in the last subsection of this article. Mutants that have lysine in position 59 also have higher protein friction, $\sigma$. This indicates that both forward and back reactions involve position 59 in the rearrangement. Mutant Tyr83Leu behaves the same as the wild-type plastocyanin. Upon substituting the values of the free energy of activation and of the protein friction in eq 15 and setting the viscosity to zero, values of $k_{0}$ in Table 2 are obtained. These values are estimates of the rate constant for the electron-transfer step, $k_{\mathrm{ET}}$, and they are comparable to the observed rate constants. Thus we cannot discount the possibility that the observed process has contributions from the electron-transfer step. Obviously, at lower viscosity the contribution from the electron-transfer step to the slow rearrangement step will be more important.

Activation Parameters. The unimolecular rate constant $k_{\mathrm{F}}$ increases as the temperature increases. Fittings of this dependence to the Eyring equation (eq 14) yielded the activation parameters $\Delta H^{\ddagger}$ and $\Delta S^{\ddagger}$. The activation enthalpy was attributed to the change in the character of the exposed surfaces in the rate-determining rearrangement (configurational fluctuation) of the diprotein complex ${ }^{3} \mathrm{Zncyt} / \mathrm{pc}$ (II) (53, 54). The negative activation entropy was attributed to the closer approach of the two protein molecules as the rearranged complex tightens for the fast electron-transfer reaction to occur $(53,54)$. The positively charged and bulky lysine residue in the pathway of rearrangement impedes the motion of the positively charged zinc cytochrome $c$ (the effect on $\Delta H^{\ddagger}$ ) and hinders its close approach to plastocyanin (the effect on $\Delta S^{\ddagger}$ ) (42).

Table 2 shows the effects of mutations in plastocyanin upon the activation parameters for the forward and back reactions. That in all cases the activation entropy is negative indicates a loss of vibrational and/or rotational freedom in the reactive configuration. Two kinds of comparisons can be done-between the forward and back reactions for each plastocyanin variant and between the back reactions for different variants. Although the values of both activation enthalpy and activation entropy are similar for the forward and back reactions, the activation enthalpy tends to be smaller and the activation entropy the same or more negative for the back reaction. Thus the smaller free energy of activation 
Table 3: Properties of Plastocyanin ${ }^{a}$

\begin{tabular}{lllcrrrr}
\hline & & & & \multicolumn{4}{c}{ angle (deg) } \\
\cline { 5 - 8 } \multicolumn{1}{c}{ pc species } & & $Z^{b}$ & $\mu^{b}(\mathrm{D})$ & $\mathrm{Cu}$ & $59^{c}$ & $60^{d}$ & $83^{e}$ \\
\hline \multirow{2}{*}{ wild type } & $\mathrm{pc}(\mathrm{II})$ & -8 & 321 & 84 & 22 & 35 & 19 \\
Glu59Lys & $\mathrm{pc}(\mathrm{I})$ & -9 & 331 & 76 & 16 & 31 & 14 \\
& $\mathrm{pc}(\mathrm{II})$ & -6 & 187 & 96 & 36 & 37 & 27 \\
Glu59Lys/Glu60Gln & $\mathrm{pc}(\mathrm{I})$ & -7 & 190 & 82 & 25 & 38 & 14 \\
& $\mathrm{pc}(\mathrm{II})$ & -5 & 144 & 108 & 56 & 69 & 40 \\
Tyr83Leu & $\mathrm{pc}(\mathrm{I})$ & -6 & 139 & 90 & 43 & 58 & 22 \\
& $\mathrm{pc}(\mathrm{II})$ & -8 & 323 & 84 & 23 & 35 & 30 \\
& $\mathrm{pc}(\mathrm{I})$ & -9 & 333 & 76 & 17 & 31 & 27 \\
\hline
\end{tabular}

${ }^{a}$ Charge $(Z)$, dipole moment $(\mu)$, and angle that the negative end of the dipole moment forms with the position vector from the center of mass to each of four selected atoms in plastocyanin are listed. ${ }^{b}$ Assuming usual $\mathrm{p} K_{\mathrm{a}}$ values at $\mathrm{pH} 7 .{ }^{c} \mathrm{C} \delta$ in residue 59 (Glu or Lys). ${ }^{d} \mathrm{C} \delta$ in residue 60 (Glu or Gln). ${ }^{e}$ Phenolic $\mathrm{C}$ in Tyr83 or C $\epsilon$ in Leu83.

can be attributed to the smaller enthalpy of activation. If the forward and the back reactions occur in the same reactive configuration of the diprotein complexes, the comparisons indicate that after the forward reaction the diprotein complex remains close to this reactive configuration. The smaller free energy of activation (and also the smaller enthalpy of activation) is required for the back reaction than was required for the forward reaction. The activation enthalpies are larger for the mutants with lysine in position 59, and from that we conclude that the observed rearrangement in both the forward and the back direction involves position 59 in plastocyanin. The positively charged and bulky Lys59 lowers the rate and increases the activation enthalpy of both the forward and the back reactions.

The steps after formation of the triplet state of zinc cytochrome $c$ are as follows. The initial binding configuration of the diprotein complex ${ }^{3} \mathrm{Zncyt} / \mathrm{pc}$ (II), in which the basic patch of cytochrome $c$ abuts the acidic patch of plastocyanin, rearranges to the reactive configuration, in which the electronic coupling between the heme and the copper site is enhanced. The forward electron transfer occurs in this reactive configuration, in which cytochrome $c$ has moved toward the copper atom and closer to the plastocyanin than in the initial binding configuration. After reduction of plastocyanin, the diprotein complex $\mathrm{Zncyt}^{+} / \mathrm{pc}(\mathrm{I})$ does not return to the initial binding configuration, because the electrostatic interactions are now different. The back reaction occurs from a reactive configuration that may be the same as, or different from, the reactive configuration of the diprotein complex ${ }^{3} \mathrm{Zncyt} / \mathrm{pc}(\mathrm{II})$.

The higher rate and the lower activation enthalpy for the back reaction are easily understood with reference to the dipole moments in Table 3. The center of the negative potential on the plastocyanin surface, as described by the dipole-moment vector, is closer to the hydrophobic patch in cuproplastocyanin than in cupriplastocyanin. The upper

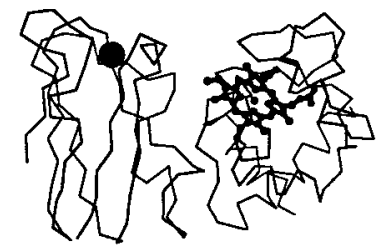

A

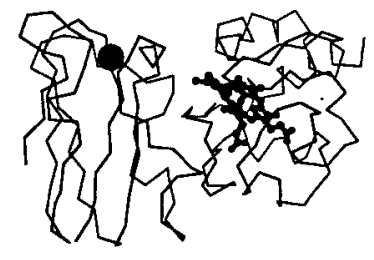

C

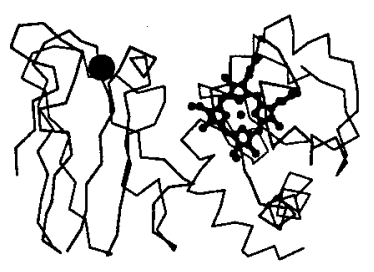

$\mathrm{B}$

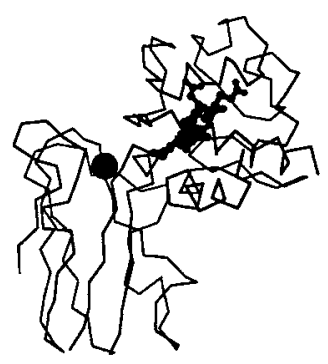

$\mathrm{D}$
FIGURE 4: Four configurations of the metalloprotein complexes between zinc cytochrome $c$ and wild-type spinach cupriplastocyanin. The configurations $\mathrm{A}-\mathrm{D}$ all have $\phi=0 ; \mathrm{A}$ has $\theta_{1}=0^{\circ}$ and $\theta_{2}=$ $180^{\circ}$; B has $\theta_{1}=30^{\circ}$ and $\theta_{2}=180^{\circ}$; C has $\theta_{1}=30^{\circ}$ and $\theta_{2}=$ $167^{\circ}$; and $\mathrm{D}$ has $\theta_{1}=30^{\circ}$ and $\theta_{2}=121^{\circ}$.

acidic cluster and the surface between this acidic cluster and the hydrophobic patch are favorable for electron transfer (66). The reduction of copper shifts the negative potential on the plastocyanin surface toward the hydrophobic patch, so that zinc cytochrome $c$ cation radical does not bind to cuproplastocyanin at the center of the acidic patch but toward the upper acidic cluster. The rearrangement to the reactive configuration, favorable for electron transfer, is thus facilitated.

Coulombic Energies of the Diprotein Complexes. The diprotein complex between cytochrome $c$ and plastocyanin is dynamic, and we measure the rate constants $k_{\mathrm{F}}$ and $k_{\mathrm{B}}$ for the rearrangements of the diprotein complexes ${ }^{3} \mathrm{Zncyt} /$ $\mathrm{pc}(\mathrm{II})$ and $\mathrm{Zncyt}^{+} / \mathrm{pc}(\mathrm{I})$. This interconversion of different configurations, which can be envisioned as configurational fluctuation, is consistent with a very recent NMR spectroscopic study (89). The Coulombic interactions are almost the same in several families of configurations in which cytochrome is docked in the general domain that contains the acidic patch of plastocyanin (24). Our calculated energies, given in Table 4, are also similar for the different configurations, shown in Figure 4. These energies are larger than the free energies of association because no correction for the ionic strength is made; the entries in Table 4 are free energies of association in water at zero ionic strength. In configuration A the Coulombic interaction is maximized $\left(\theta_{1}=0^{\circ}\right.$ and $\theta_{2}$ $\left.=180^{\circ}\right)$; in $\mathrm{B}$ the exposed heme edge in cytochrome $c\left(\theta_{1}\right.$ $=30^{\circ}$ ) abuts the center of the acidic patch in plastocyanin

Table 4: Coulombic Energies (E) in Four Configurations of the Diprotein Complexes Zncyt/Pc(II) and Zncyt ${ }^{+} / \mathrm{Pc}(\mathrm{I})$

\begin{tabular}{|c|c|c|c|c|c|c|c|c|}
\hline \multirow[b]{3}{*}{ pc species } & \multicolumn{8}{|c|}{$E(\mathrm{~kJ} / \mathrm{mol})$} \\
\hline & \multicolumn{4}{|c|}{ Zncyt/pc(II) } & \multicolumn{4}{|c|}{$\mathrm{Zncyt}^{+} / \mathrm{pc}(\mathrm{I})$} \\
\hline & A & B & $\mathrm{C}$ & $\mathrm{D}$ & A & B & $\mathrm{C}$ & $\mathrm{D}$ \\
\hline wild type & -70.7 & -67.6 & -66.5 & -55.5 & -87.9 & -84.3 & -83.1 & -70.4 \\
\hline Glu59Lys & -49.3 & -47.2 & -46.6 & -40.2 & -63.2 & -60.7 & -60.0 & -52.7 \\
\hline Glu59Lys/Glu60Gln & -40.3 & -38.6 & -38.1 & -33.2 & -52.3 & -50.2 & -49.7 & -44.4 \\
\hline Tyr83Leu & -70.8 & -67.6 & -66.6 & -55.5 & -88.0 & -84.4 & -83.2 & -70.4 \\
\hline
\end{tabular}


$\left(\theta_{2}=180^{\circ}\right)$, an area unfavorable for electron-transfer; and $\mathrm{C}$ and $\mathrm{D}$ are two plausible configurations in which both plastocyanin $\left(\theta_{2}=167^{\circ}\right.$ and $\theta_{2}=121^{\circ}$, respectively) and cytochrome $c\left(\theta_{1}=30^{\circ}\right)$ are oriented favorably for electron transfer. The angles $\theta_{2}$ in configurations $\mathrm{C}$ and $\mathrm{D}$ correspond to the atoms in residues 59 and 88 on the plastocyanin surface residues that are involved in the rearrangement. As expected, the complexes containing the mutants Glu59Lys and Glu59Lys/Glu60Gln have lower Coulombic interaction (less negative values of $E$ ) than the complexes containing wildtype plastocyanin and the mutant Tyr83Leu. For the diprotein complex ${ }^{3} \mathrm{Zncyt} / \mathrm{pc}$ (II) the respective values are -33 to -49 vs -56 to $-71 \mathrm{~kJ} / \mathrm{mol}$, and for the diprotein complex $\mathrm{Zncyt}^{+} /$ $\mathrm{pc}(\mathrm{I})$ the values are -44 to $-63 \mathrm{vs}-70$ to $-88 \mathrm{~kJ} / \mathrm{mol}$. Coulombic energies are somewhat more negative for $\mathrm{Zncyt}^{+} /$ pc(I) than for ${ }^{3} \mathrm{Zncyt} / \mathrm{pc}$ (II) because both monopolar and dipolar interactions are larger in the former complex. This indication that the protein association is stronger for the back reaction than for the forward reaction justifies the approximation made in eq 7 that the back reaction is purely unimolecular. The difference between the interactions in the diprotein complexes $\mathrm{Zncyt}^{+} / \mathrm{pc}$ (I) and ${ }^{3} \mathrm{Zncyt} / \mathrm{pc}$ (II) is smaller for Glu59Lys and Glu59Lys/Glu60Gln than for wild-type plastocyanin and Tyr83Leu. For example, the rearrangement of $\mathrm{Zncyt}^{+} / \mathrm{pc}(\mathrm{I})$ from configuration $\mathrm{B}$ to D requires $6 \mathrm{~kJ} /$ mol for Glu59Lys/Glu60Gln and $14 \mathrm{~kJ} / \mathrm{mol}$ for wild-type plastocyanin; and the rearrangement of ${ }^{3} \mathrm{Zncyt} / \mathrm{pc}$ (II) from configuration $\mathrm{B}$ to $\mathrm{D}$ requires $5 \mathrm{~kJ} / \mathrm{mol}$ for Glu59Lys/ Glu60Gln and $12 \mathrm{~kJ} / \mathrm{mol}$ for wild-type plastocyanin. (These values are calculated differences in stability of configurations in Table 4.) The mutant Glu59Lys/Glu60Gln easily rotates around $\theta_{2}$ and has shallower Coulombic-potential surface than wild-type plastocyanin. Thus it is not surprising that for Glu59Lys the free energies of activation for the forward and back reaction are 47.2 and $45.9 \mathrm{~kJ} / \mathrm{mol}(1.3 \mathrm{~kJ} / \mathrm{mol}$ difference), and for wild-type plastocyanin free energies of activation for the forward and back reaction are 40.8 and $37.4 \mathrm{~kJ} / \mathrm{mol}(3.4 \mathrm{~kJ} / \mathrm{mol}$ difference $)$.

\section{CONCLUSION}

In many photoinduced electron-transfer reactions the excited-state forward reaction is very rapidly followed by the ground-state back reaction; the two processes are often difficult to separate and examine. In this study, fortunately, the back reaction is only a little faster than the forward reaction. We were able to obtain not only the rate constants but also the thermodynamic activation parameters for both reactions, involving zinc cytochrome $c$ and several variants of plastocyanin. Kinetic effects of solution viscosity and simple theoretical considerations of Coulombic interactions between associated metalloproteins threw light on the dynamic processes (rearrangement of the diprotein complexes) by which both the forward and the back reaction are gated.

\section{ACKNOWLEDGMENT}

We thank Professor James H. Espenson and Dr. Andreja Bakac for allowing us to use their Nd-YAG laser and for their help with it. G.M.U. thanks Boehringer Ingelheim Fonds for a fellowship.

\section{REFERENCES}

1. Brunschwig, B. S., and Sutin, N. (1989) J. Am. Chem. Soc. $111,7454$.

2. Hoffman, B. M., and Ratner, M. A. (1987) J. Am. Chem. Soc $109,6237$.

3. Hoffman, B. M., Ratner, M. A., and Wallin, S. A. (1990) $A d v$ Chem. Ser. 226, 125.

4. Hoffman, B. M., and Ratner, M. A. (1988) J. Am. Chem. Soc. $110,8267$.

5. Nocek, J. M., Stemp, E. D. A., Finnegan, M. G., Koshy, T. I., Johnson, M. K., Margoliash, E., Mauk, A. G., Smith, M., and Hoffman, B. M. (1991) J. Am. Chem. Soc. 113, 6822.

6. Fietelson, J., and McLendon, G. (1991) Biochemistry 30, 5051.

7. Walker, M. C., and Tollin, G. (1992) Biochemistry 31, 2798.

8. Sullivan, E. P., Jr., Hazzard, J. T., Tollin, G., and Enemark, J. H. (1992) J. Am. Chem. Soc. 114, 9662.

9. Davidson, V. L. (1996) Biochemistry 35, 14035.

10. Sykes, A. G. (1991) Adv. Inorg. Chem. 36, 377

11. Sykes, A. G. (1991) Struct. Bonding 75, 177

12. Moore, G. R., and Pettigrew, G. W. (1990) Cytochrome c: Evolutionary, Structural, and Physiochemical Aspects, SpringerVerlag, Berlin.

13. Moore, G. R., Williams, R. J. P., Chien, J. C. W., and Dickinson, L. C. (1980) J. Inorg. Biochem. 13, 1.

14. Scott, R. A., and Mauk, A. G., Ed. (1996) Cytochrome c: A Multidisciplinary Approach, University Science Books, Sausalito, CA.

15. Anni, H., Vanderkooi, J. M., and Mayne, L. (1995) Biochemistry 34,5744

16. Angiolillo, P. J., and Vanderkooi, J. M. (1995) Biophys. J. $68,2505$.

17. Vanderkooi, J. M., and Erecińska, M. (1975) Eur. J. Biochem. 60, 199.

18. Vanderkooi, J. M., Adar, F., and Erecińska, M. (1976) Eur. J. Biochem. 64, 381.

19. Vanderkooi, J. M., Landesberg, R., Haydon, G., and Owen, C. (1977) Eur. J. Biochem. 81, 339

20. Erecińska, M., and Vanderkooi, J. M. (1978) Methods Enzymol. $53,165$.

21. Ye, S., Shen, C., Cotton, T. M., and Kostić, N. M. (1997) J. Inorg. Biochem. 65, 219.

22. King, J. C., Binstead, R. A., and Wright, P. E. (1985) Biochim. Biophys. Acta 106, 262.

23. Bagby, S., Driscoll, P. C., Goodall, K. G., Redfield, C., and Hill, H. A. O. (1990) Eur. J. Biochem. 188, 413.

24. Roberts, W. A., Freeman, H. C., Getzoff, E. D., Olson, A. J., and Tainer, J. A. (1991) J. Biol. Chem. 266, 13431.

25. Geren, L. M., Stonehuerner, J., Davies, D. J., and Millett, F. (1983) Biochim. Biophys. Acta 724, 62.

26. Zhou, J. S., and Kostić, N. M. (1992) Biochemistry 31, 7543.

27. Nordling, M., Sigfridsson, K., Young, S., Lundberg, L. G., and Hansson, Ö. (1991) FEBS Lett. 291, 327.

28. Modi, S., He, S., Gray, J. C., and Bendall, D. S. (1992) Biochim. Biophys. Acta 1101, 64.

29. Modi, S., Nordling, M., Lundberg, L. G., Hansson, Ö., and Bendall, D. S. (1992) Biochim. Biophys. Acta 1102, 85.

30. Sigfridsson, K., Young, S., and Hansson, Ö. (1996) Biochemistry 35, 1249.

31. Sigfridsson, K., Young, S., and Hansson, Ö. (1997) Eur. J. Biochem. 245, 805.

32. Young, S., Sigfridsson, K., Olesen, K., and Hansson, Ö. (1997) Biochim. Biophys. Acta 1322, 106.

33. Haehnel, W., Jansen, T., Gause, K., Klögsgen, R. B., Stahl, B., Michl, D., Huvermann, B., Karas, M., and Herrmann, R. G. (1994) EMBO J. 13, 1028 .

34. Hippler, M., Reichert, J., Sutter, M., Zak, E., Altschmied, L. Schröer, U., Hermann, R. G., and Haehnel, W. (1996) EMBO J. 15,6374 .

35. Díaz, A., Hervás, M., Navarro, J. A., De la Rosa, M., and Tollin, G. (1994) Eur. J. Biochem. 222, 1001.

36. Hervás, M., Navarro, J. A., Díaz, A., and De la Rosa, M. (1996) Biochemistry 35, 2693.

37. De la Cerda, B., Navarro, J. A., Hervás, M., and De la Rosa, M. (1997) Biochemistry 36, 10125. 
38. He, S., Modi, S., Bendall, D. S., and Gray, J. C. (1991) EMBO J. 10, 4011.

39. Lee, B. H., Hibino, T., Takabe, T., and Weisbeek, P. J. (1995) J. Biochem. (Tokyo) 117, 1209.

40. Kannt, A., Young, S., and Bendall, D. S. (1996) Biochim. Biophys. Acta 1277, 115.

41. Crnogorac, M. M., Shen, C., Young, S., Hansson, Ö., and Kostić, N. M. (1996) Biochemistry 35, 16465.

42. Ivković-Jensen, M. M, Ullmann, G. M., Young, S., Hansson, Ö., Crnogorac, M. M., Ejdebäck, M., and Kostić, N. M. (1998) Biochemistry 37, 9557.

43. Zhou, J. S., and Kostić, N. M. (1991) J. Am. Chem. Soc. 113, 6067.

44. Zhou, J. S., and Kostić, N. M. (1991) J. Am. Chem. Soc. 113, 7040 .

45. Zhou, J. S., and Kostić, N. M. (1992) J. Am. Chem. Soc. 114, 3562.

46. Zhou, J. S., and Kostić, N. M. (1992) Spectrum 5 (2), 1.

47. Zhou, J. S., Brothers, H. M., II, Neddersen, J. P., Peerey, L. M., Cotton, T. M., and Kostić, N. M. (1992) Bioconjugate Chem. 3, 382.

48. Zhou, J. S., and Kostić, N. M. (1993) Biochemistry 32, 4539.

49. Zhou, J. S., and Kostić, N. M. (1993) J. Am. Chem. Soc. 115, 10796.

50. Qin, L., and Kostić, N. M. (1996) Biochemistry 35, 3379.

51. Kostić, N. M. (1996) in Metal-Containing Polymeric Materials (Pitman, C. U., Jr., et al., Eds.) Plenum, New York.

52. Qin, L., and Kostić, N. M. (1994) Biochemistry 33, 12592.

53. Ivković-Jensen, M. M., and Kostić N. M. (1996) Biochemistry $35,15095$.

54. Ivković-Jensen, M. M., and Kostić N. M. (1997) Biochemistry 36,8135 .

55. Wendoloski, J. J., Matthew, J. B. Webber, P. C., and Salemme, F. R. (1987) Science 238, 794.

56. Northrup, S. H., Boles, J. O., and Reynolds, J. C. L. (1988) Science 241, 67.

57. Rodgers, K. K., Pochapsky, T. C., and Sligar, S. G. (1988) Science 240, 1657.

58. Burch, A. M., Rigby, S. E. J., Funk, W. D., MacGilliwray, R. T. A., Mauk, M. R., Mauk, A. G., and Moore, G. R. (1990) Science 247, 831.

59. Wallin, S. A., Stemp, E. D. A., Everest, A. M., Nocek, J. M., Netcel, T. L., and Hoffman, B. M. (1991) J. Am. Chem. Soc. 113,1842

60. McLendon, G., Zhang, Q., Wallin, S. A., Miller, R. M., Billestone, W., Spears, K. G., and Hoffman, B. M. (1993) J. Am. Chem. Soc. 115, 3665.

61. Kostić, N. M. (1991) Met. Ions Biol. Syst. 27, 129.

62. Chen, L., Poliks, R., Hamada, K., Chen, Z., Mathews, F. S., Davidson, V. L., Satow, Y., Huizinga, E., Vellieux, F. M. D., and Hol, W. G. J. (1992) Biochemistry 31, 4959.

63. Willie, A., Steyton, P. S., Sligar, S. G., Durham, B., and Millett, F. (1992) Biochemistry 31, 7237.

64. Chen, L., Durley, R. C. E., Mathews, F. S., and Davidson, V. L. (1994) Science 264, 86.
65. Mauk, M. R., Ferrer, J. C., and Mauk, A. G. (1994) Biochemistry 33, 12609.

66. Ullmann, G. M., and Kostić, N. M. (1995) J. Am. Chem. Soc. $117,4766$.

67. Ullmann, G. M., Knapp, E. W., and Kostić, N. M. (1997) J. Am. Chem. Soc. 119, 42.

68. Connolly, M. L. (1983) Science 221, 709.

69. CRC Handbook of Chemistry and Physics (1986) (Weast, R. C., Ed.) 66th ed., CRC Press, Boca Raton, FL.

70. CRC Handbook of Biochemistry and Molecular Biology (1975) 3rd ed., CRC Press, Cleveland, OH

71. Miner, C. S., and Dalton, N. N. (1953) Glycerol, Reinhold, New York.

72. Ejdebäck, M., Young, S., Samuelsson, A., and Karlsson, B. G. (1997) Protein Expression Purif. 11, 17.

73. Huston, P., Espenson, J. H., and Bakac, A. (1992) J. Am. Chem. Soc. 114, 9510.

74. Bushnell, G. W.; Louie, G. V., and Brayer, G. D. (1990) J. Mol. Biol. 214, 585.

75. Guss, J. M., Bartunik, H. D., and Freeman, H. C. (1992) Acta Crystallogr., Sect. B 48, 790.

76. Brooks, B. R., Bruccoleri, R. E., Olafson, B. D., States, D. J., Swaminathan, S., and Karplus, M. (1983) J. Comput. Chem. 4, 187.

77. Koppenol, W. H., and Margoliash, E. (1982) J. Biol. Chem. 257,4426

78. MacKerell, A. D., Bashford D., Bellott, M., Dunbrack, R. L., Jr., Evanseck, J. D., Field, M. J., Fischer, S., Gao, J., Guo, H., Ha, S., Joseph-McCarthy, D., Kuchnir, L., Kuczera, K., Lau, F. T. K., Mattos, C., Michnick, S., Ngo, T., Nguyen, D. T., Prodhom, B., Reiher, W. E., Roux, B., Schlenkrich, M., Smith, J. C., Stote, R., Straub, J., Watanabe, M., WiorkiewiczKuczera, J., Yin, D., and Karplus, M. (1998) J. Phys. Chem. B 102, 3586

79. Kabsch, W. (1978) Acta Crystallogr. A 32, 922.

80. Stone, A. J. (1996) The Theory of Intermolecular Forces, Clarendon Press, Oxford, England.

81. Taniguchi, V. T., Sailasuta-Scott, N., Anson, F. C., and Gray, H. B. (1980) Pure Appl. Chem. 52, 2275.

82. Ortega, J. M., Mathis, P., and Williams, J. C. (1996) Biochemistry 35, 3354.

83. Scott, J. R., Willie, A., and McLean, M. (1993) J. Am. Chem. Soc. 115,6820 .

84. Beece, D., Eisenstein, L., Frauenfelder, H., Good, D., Mardem, M. C., Reinisch, L., Reynolds, A. H., Sorensen, L. B., and Yue, K. T. (1980) Biochemistry 19, 5147.

85. Gavish, B., and Werber, M. M. (1979) Biochemistry 18, 1269.

86. Khoshtariya, D. E., Hammerstad-Pedersen, J. M., and Ulstrup, J. (1991) Biochim. Biophys. Acta 1076, 359

87. Ansari, A., Jones, C. M., Henry, E. R., Hofrichter, J., and Eaton, W. A. (1992) Science 256, 1796.

88. Kramers, H. A.(1940) Physica (Utrecht) 7, 284.

89. Ubbink, M., and Bendall, D. S. (1997) Biochemistry 36, 6326. BI9817156 